University of Louisville

ThinkIR: The University of Louisville's Institutional Repository

\title{
Targeting the anaphase promoting complex/cyclosome to induce mitotic arrest in ovarian cancer.
}

Douglas John Saforo

University of Louisville

Follow this and additional works at: https://ir.library.louisville.edu/honors

Part of the Biology Commons

\section{Recommended Citation}

Saforo, Douglas John, "Targeting the anaphase promoting complex/cyclosome to induce mitotic arrest in ovarian cancer." (2013). College of Arts \& Sciences Senior Honors Theses. Paper 22.

http://doi.org/10.18297/honors/22

This Senior Honors Thesis is brought to you for free and open access by the College of Arts \& Sciences at ThinkIR: The University of Louisville's Institutional Repository. It has been accepted for inclusion in College of Arts \& Sciences Senior Honors Theses by an authorized administrator of ThinkIR: The University of Louisville's Institutional Repository. This title appears here courtesy of the author, who has retained all other copyrights. For more information, please contact thinkir@louisville.edu. 
Targeting the Anaphase Promoting Complex/Cyclosome to induce Mitotic Arrest in Ovarian Cancer

By

Douglas John Saforo

Submitted in partial fulfillment of the requirements

for Graduation summa cum laude

and

for Graduation with Honors from the Department of Biology

University of Louisville

May, 2013 


\section{Abstract}

Taxanes are a class of chemotherapeutic drug that act to disrupt microtubule function and cause mitotic arrest and cell death. These drugs are commonly used for cancer treatment, however their effectiveness is dependent on the presence of a functioning spindle assembly checkpoint (SAC). As a result, it is possible for cancer cells to become resistant to microtubule disrupting drugs by inactivating the SAC. The anaphase promoting complex/cyclosome $(\mathrm{APC} / \mathrm{C})$ is an E3 ubiquitin ligase that acts as the master regulator of cell cycle progression. Inhibition of the APC/C should result in disruption of the cell cycle, resulting in arrest during mitosis and/or pseudo-G1. Previous in silico studies of the APC/C structure identified compounds that potentially bind to the APC/C subunit ANAPC2. Three of these compounds (\#8, 10, and 11) were tested on A2780/CP70 and SKOV3 ovarian carcinoma cells and tGM24 telomerase immortalized human fibroblasts. Cell morphology was observed during treatment with the compounds and showed signs of mitotic and apoptotic cells in a dose dependent manner. While all compounds showed the predicted effects, effects were more pronounced with compounds 10 and 11 than compound 8. Mitotic index determinations revealed a significant mitotic delay in both cancer cells and fibroblasts treated with compounds 8,10 , and 11. Compounds 10 and 11 were more potent than compound 8 in inhibition of colony forming ability for all three cell lines. Fibroblasts showed some resistance to compound 8, however fibroblasts exposed to compound 11 showed complete inhibition of cell growth without characteristic morphological signs of apoptosis. All three compounds induced apoptosis in ovarian cancer cells, as indicated by increased caspase 3 activity, but not in fibroblasts. These results indicate that compounds targeting the APC/C can induce mitotic arrest and kill ovarian cancer cells while sparing normal cells. 


\section{Introduction}

Ovarian cancer accounts for more deaths than any other cancer of the female reproductive system and is the fourth deadliest cancer afflicting women overall. In the United States, it is estimated that 22,280 women will be diagnosed with ovarian cancer and that 15,500 will die from the disease in 2012 [1]. These statistics have endured over the past twenty years. Despite this, improvements in the effectiveness of surgery and fine tuning of the administration of cytotoxic compounds have led to a higher survival rate of patients overall, and particularly those suffering from later stages of the disease. Patients suffering from stage I ovarian cancer, while the cancer is still confined within the ovary, have shown a five year survival rate of between 70 and $90 \%$ after treatment. The rate of survival vastly decreases once the cancer has progressed to stage II and spread to the pelvic organs. As the cancer progresses to the peritoneal cavity and beyond, stage III and IV, rate of survival drops below $30 \%$.

Early detection of ovarian cancer is thus highly important, but has posed a challenging problem for physicians. It is not uncommon for symptoms associated with the onset of disease being similar to other more common ailments. These symptoms include abdominal bloating and pain, indigestion, urinary frequency, constipation, and other abdominal and gastrointestinal symptoms [2]. Patients were reported to have believed these symptoms were due to factors such as menopause or stress, resulting in a significant delay in procuring a proper diagnosis and treating the disease while still manageable. This delay adds further difficulty to treatment and decreases the likelihood of survival, as only $20 \%$ of patients are diagnosed during stage I [3]. While screening techniques such as transvaginal sonography and serum markers are actively being researched [4], until better screening techniques are developed and made financially viable, the optimization and refinement of current treatments provides the best means of increasing the survival rate of patients suffering from later stages of ovarian cancer progression. Development of more effective treatments may serve to decrease the risk 
recurrence of disease in those patients diagnosed with ovarian cancer and successfully treated in stage I.

Biologically, a majority of the most deadly ovarian cancers can be divided into several different categories depending upon their histotypes. These subtypes include: high grade serous carcinomas, endometrioid carcinomas, clear cell carcinomas, mucinous carcinomas, and low grade serous carcinomas. While all are classified as ovarian cancer, each of these subtypes are pathologically distinct and display differences in risk factors, metastasis, oncogenesis, and response to chemotherapy [5]. It is primarily believed that these cancers originate due to metaplasia of the surface cells covering the ovary and neoplasms implanted on the peritoneal membrane, however evidence also suggests that many of these different subtypes of carcinoma are derived from locations outside of the ovary, including the fallopian tube and endometrium [6]. This broad range of disease highlights the need for treatments that target molecules necessary for proliferation in a wide variety of cell types.

Currently, ovarian cancers are treated with a combination of surgery and chemotherapeutics [7]. Cytoreductive surgery, either removing tumors completely or sparing those less than a centimeter in diameter, has long been a staple in treatment of early and late stage ovarian cancers [8]. In a clinical study, patients who were unable to undergo cytoreductive therapy and instead underwent only chemotherapy had a substantially lower rate of remission, and those that did respond to treatment were disease free for a period between seven to eleven months [9]. The combination of chemotherapy and cytoreductive surgery has been responsible for the increase in survival of later stage ovarian cancers and is regarded as the optimum treatment for managing the disease at all stages [10]. Currently, the standard chemotherapy treatment involves the intravenous administration of drugs followed by an intensive regimen of concentrated drugs intraperitoneally. These drugs are typically a combination of platinum containing compounds, such as cisplatin, and taxanes. 
Taxanes, such as Taxol® or paclitaxel, are a class of drugs that has been shown to be effective against ovarian cancer [11]. Originally derived from the Pacific yew tree, paclitaxel acts as a mitotic inhibitor and disrupts the normal function of microtubules during cell division, leading to mitotic arrest and apoptosis. Microtubules are cellular structures important for a variety of tasks and processes essential to cell function. They are involved in creating and maintaining cell shape, cell motility, intracellular transport, cell division, and mitosis. The microtubule structure is that of a tube shaped polymer of proteins that can be rapidly assembled into long filaments and disassembled to component monomers. This dynamic structure is tightly regulated and directed by a number of microtubule-regulator proteins. One of the essential tasks of the cellular microtubule in eukaryotic cells is the movement and segregation of chromosomes during mitosis. This function is highly dependent on the rapid polymerization and depolymerization of microtubules, allowing the connection of mitotic spindle fibers to kinetochores on the chromosome, the subsequent alignment of chromosomes along the metaphase plate, and the separation and segregation of chromosomes during anaphase.

Paclitaxel is classified as a microtubule stabilizer. Its mechanism of action is believed to involve binding to chains of tubulin molecules and inducing a conformational change which results in increased affinity of free tubulin molecules for the tubulin chain, thus resulting in an increase in polymerization. Cells that have been treated with paclitaxel and enter into mitosis are unable to properly align chromosomes along the metaphase plate during pro-metaphase. The spindle assembly checkpoint (SAC) monitors the proper attachment of mitotic spindle fibers to kinetochores by sensing microtubule tension to prevent the premature separation of sister chromatids before they are properly aligned during pro-metaphase. The proteins and enzymes that participate in the SAC are spatially located near the kinetochore. These proteins include mitotic arrest deficient proteins, MAD1 and MAD2, budding uninhibited by benzimidazole proteins, BUBR1 and BUB3, and also TTK protein kinase [12]. Collectively, these proteins act to prevent the activity of the anaphase promoting complex/ cyclosome (APC/C), which is an E3 
ubiquitin ligase responsible for the tagging of proteins for proteolysis. This mechanism of inhibition is achieved by sequestering the APC/C coactivator cell division cycle protein 20 (CDC20) until the spindle fibers that are extending out from centrioles at polar sides of the cell have properly attached to kinetochore structures on the centromeric region of the chromosomes and the chromosomes have been properly arranged along the medial region of the cell. This is a very tightly regulated and delicate process, and the improper attachment of a single chromosome has been shown to be sufficient to activate the SAC and delay progression through mitosis. Once the requirements of the SAC are met, CDC20 is released and the APC/C is able to proceed with its function [13].

Paclitaxel is able to maintain the SAC by inhibiting the proper attachment of spindle fibers to kinetochores, causing cells to arrest in mitosis. Cancer cells that are subjected to a prolonged mitotic arrest are unable to recover and consequently undergo apoptosis (programmed cell death) [14]. Thus, in cells with a functional SAC, paclitaxel has proven itself as a powerful anti-cancer drug. However, maintenance of a functioning SAC is not essential in tumorigenesis and uncontrolled cell proliferation, and some cancers can thrive while not having a functioning SAC. This is just one scenario in which cancers can become resistant to paclitaxel therapy. Another scenario involves cancers that utilize mutant tubulin proteins that are less prone to having their polymerization amplified by paclitaxel binding [15]. Cancers that have established resistance to paclitaxel and cisplatin are a growing concern. Since drugs which act by interrupting mitosis have shown such efficacy as anti-cancer agents, this resistance to current chemotherapeutics highlights a need for new drug treatments that similarly can cause mitotic arrest but by a mechanism other than that utilized by paclitaxel.

One such protein that plays a vital role in the progression through mitosis and could serve as a novel target is the APC/C. The APC/C is a multi-subunit complex and a unique E3 ubiquitin ligase that acts as the master regulator of the cell cycle [16]. The APC/C is responsible for ubiquitinating a number of different substrate targets at different stages during 
the cell cycle, signaling them for degradation by the 26S proteasome [17]. Among these targets, the current understanding is that the targets most relevant to mitosis are the regulatory protein securin and the B-type cyclin (cyclin B1). Once the chromosomes have become properly aligned along the metaphase plate during prometaphase and Cdc20 is released by the SAC, the Cdc20 acts as a cofactor for the APC/C, binding and activating it. This bound APC/CCdc20 complex increases the ability for binding of securin and cyclin B1, allowing them to be polyubiquitinated, thus targeting them for degradation. Securin is a small protein that is responsible for inhibiting the activity of separase. The activity of separase, an endopeptidase, is to cleave cohesin, which holds the sister chromatids together during prophase and metaphase. Loss of cohesin allows the separation of the chromatids and entry into anaphase. Separase is also inhibited by the activity of cyclin dependent kinase 1(CDK1) [18]. CDK1 and its cofactor cyclin B1 promote the phosphorylation of an inhibitory phosphate binding site on the separase enzyme. Once cyclin B1 is targeted for degradation, the kinase activity of CDK1 is inhibited and the separase enzyme becomes dephosphorylated. This phosphatase mechanism is not completely understood, however inactivation of CDK1 relieves inhibition on the separase enzyme and promotes the cleavage of cohesin and progression to anaphase (Figure 1). 


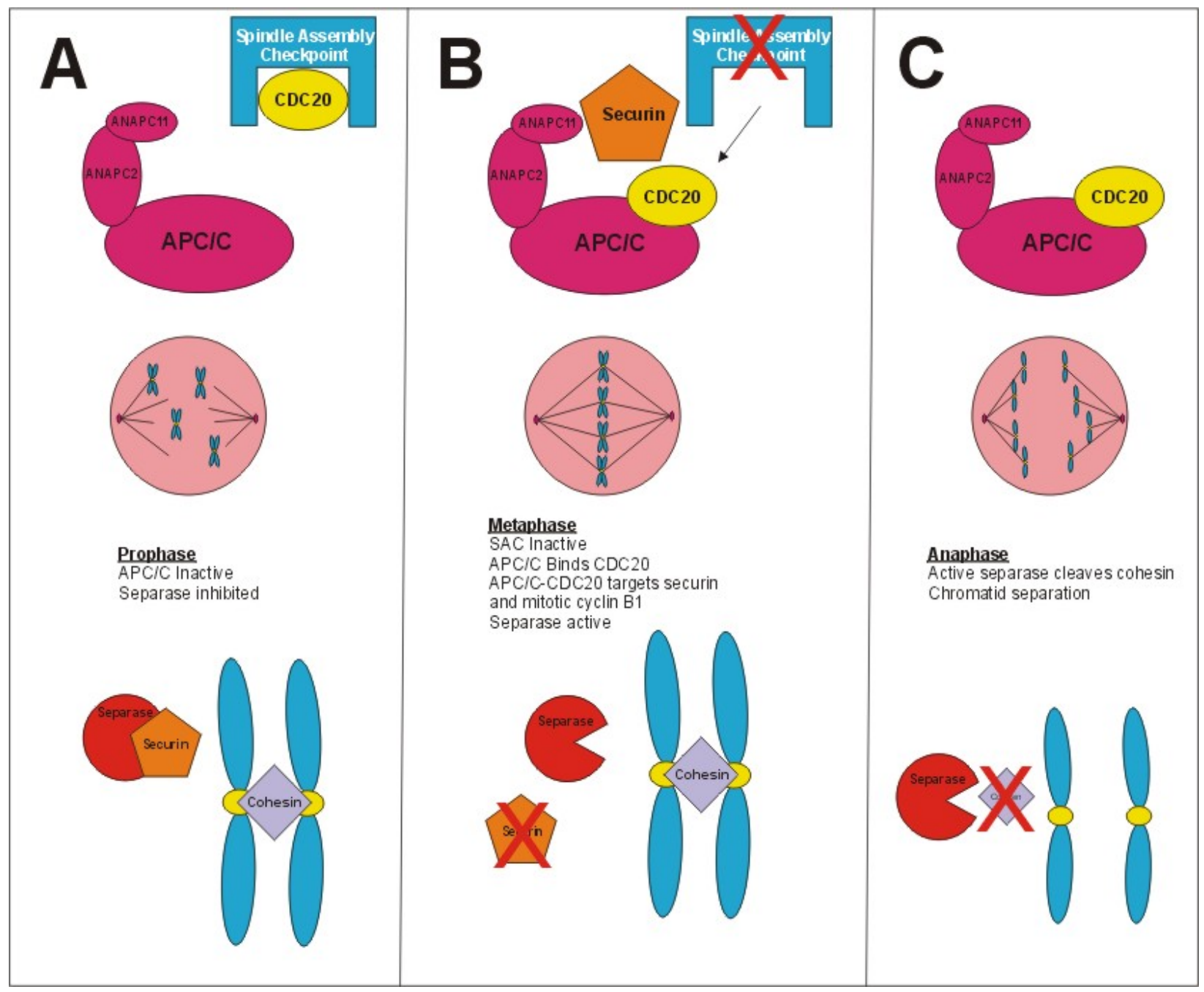

Figure 1. A simplified model of the interaction between the spindle assembly checkpoint and the APC/C during mitosis.

A. In late prophase, the CDC20 co-activator of the APC/C is sequestered by the spindle assembly checkpoint. Pairs of sister chromatids are held together by cohesin. Separase enzyme is inhibited by securin.

B. In metaphase, pairs of sister chromatids have been placed along the metaphase plate by spindle fibers. The requirements of the spindle assembly checkpoint are met, and the CDC20 co-activator is released. CDC-20 binds to the APC/C, enabling it to target securin for degradation. Separase enzyme is activated.

C. In anaphase, the active separase enzyme cleaves the cohesin protein. This allows separation of the sister chromatids, which begin to move toward polar regions of the dividing cell. 
Another $\mathrm{APC} / \mathrm{C}$ cofactor essential to cell cycle progression is the fizzy related protein homolog FZR1 (also referred to as $\mathrm{CDH} 1$ in literature). The activity of the APC/C-CDH1 complex is responsible for the modulation of levels of a number of different proteins important for the exit from mitosis and entry into $\mathrm{G} 1$. These proteins include cyclins $B 1$ and $A 2$, the origin of replication recognition complex subunit 1 (ORC1), cell division cycle 6 protein (CDC6), and geminin. ORC1, CDC6, and geminin act to regulate pre-replication complex formation during onset of G1 prior to initiating replication of DNA [19]. Degradation of cyclins results in inhibition of CDK activity, a requirement for formation of pre-replication complexes. Once pre-replication complexes have been formed on DNA, the APC/C mediates a shift from low to high CDK activity and DNA replication proceeds in S phase.

Inhibition of the APC/C allows for two clear times during the cell cycle during which the cell can undergo cell cycle arrest. The first opportunity for arrest is during the metaphase to anaphase transition of mitosis, when the APC/C is required for the proper separation of sister chromatids at the metaphase plate. The second opportunity for arrest is just prior to the transition into $\mathrm{G} 1$, where the $\mathrm{APC} / \mathrm{C}$ is required for the formation of pre-replication complexes.

The structure of the APC/C is that of a large, thirteen subunit holoenzyme organized into an asymmetric triangle structure with an outer wall and inner cavity. These subunits are organized into four main parts including a structural arm, catalytic arm, tetratricopeptide repeat arm, and stabilizers. The APC subunit 2 (ANAPC2) is a part of the catalytic arm structure and its cullin domain, which acts as the scaffold for ubiquitin ligases, lies on the outside of the structure. APC subunit 11 (ANAPC11) is a zinc RING finger protein that is tightly bound to ANAPC2 [20]. It is possible that a small targeted compound could bind to the cullin domain of ANAPC2 and prevent the association of ANAPC11 and thus inhibit function of the APC/C holoenzyme.

Previous in silico studies using a virtual screen performed at the University of Louisville James Graham Brown Cancer Center Molecular Modeling Facility analyzed the APC/C structure 
and yielded potential compounds that bind to APC/C subunits and may inhibit formation of the holoenzyme [21]. Two sites were targeted on the ANAPC2 subunit, and around 50 candidate compounds were predicted to target each site (Figure 2). The aim of this thesis is to test the hypothesis that if candidate compounds block the binding of the subunits of the APC/C and inhibit its function, then ovarian cancer cells with a functioning SAC will either undergo mitotic arrest and apoptosis or proceed to early G1, arrest, and undergo apoptosis or mitotic catastrophe as was observed in cells lacking a functional SAC [22]. To that end, three candidate compounds (compounds 8,10 , and 11) were selected to be tested for their ability to induce mitotic arrest, cytotoxicity, and induce apoptosis in two cisplatin resistant ovarian carcinoma cell lines (A2780/CP70, SKOV3), and telomerase immortalized diploid fibroblasts (tGM24). 


\section{Materials and Methods}

Cells and culture conditions. Hyclone Minimum Essential Medium alpha liquid medium, Hyclone RPMI 1640 liquid medium, Hyclone McCoy's 5A liquid medium, and Hyclone Fetal Bovine sera were obtained from Thermo Scientific. Ovarian carcinoma cells A2780/CP70 were the kind gift of Dr. Eddie Reed. Ovarian carcinoma cells SKOV3 were purchased from ATCC. GM00024 cells were originally obtained from the Coriell Cell Repository, immortalized with a retrovirus containing hTERT cDNA, and renamed tGM24 [23]. tGM24 cells were cultured in Hyclone Alpha Minimum Essential Medium supplemented with 10\% fetal bovine serum, $100 \mathrm{U} / \mathrm{ml}$ penicillin, and $10 \mathrm{U} / \mathrm{ml}$ streptomycin. A2780/CP70 cells were cultured in Hyclone RPMI 1640 medium supplemented with $10 \%$ fetal bovine serum, $100 \mathrm{U} / \mathrm{ml}$ penicillin, $10 \mathrm{U} / \mathrm{ml}$ streptomycin, and 2 mM L-glutamine (200mM stock). SKOV3 cells were cultured in Hyclone McCoy's 5A medium supplemented with $10 \%$ fetal bovine serum, $100 \mathrm{U} / \mathrm{ml}$ penicillin, $10 \mathrm{U} / \mathrm{ml}$ streptomycin, and $1.5 \mathrm{mM} \mathrm{L-glutamine.} \mathrm{All} \mathrm{cell} \mathrm{lines} \mathrm{were} \mathrm{incubated} \mathrm{at} 37^{\circ} \mathrm{C}$ in an atmosphere of $5 \% \mathrm{CO}_{2}$. Visual assessment of Morphology. Ovarian cancer cells (A2780/CP70 and SKOV3) were plated in a 24-well plate, treated with compounds at a range of concentrations (see below), and observed with a microscope over a 24 hour period with pictures taken at 6 hour intervals. Diploid fibroblasts (tGM24) were treated with candidate compounds over a 72 hour period with pictures taken at 24 hour intervals. Compound 8 was tested at concentrations including 0.4 , $0.7,1,3,5,10,20,40,60$, and $80 \mu \mathrm{M}$. Compounds 10 and 11 were tested at concentrations including $0.4,0.7,1,3,5,10,20$, and $40 \mu \mathrm{M}$. All drugs were dissolved in DMSO to stocks of 100 $\mathrm{mM}$, and dilutions to the appropriate concentrations were made from those stocks. All cell lines were also treated with paclitaxel as a positive control and DMSO (0.1\% v/vol) as a negative control. 
Cytotoxicity Assays. Quantitative determination of cytotoxicity of candidate compounds was measured using colony forming assay. Ovarian cancer cells (A2780/CP70 and SKOV3) and diploid fibroblasts (tGM24) were plated at 100 or 500 cells per well on a six well plate and allowed to attach overnight. Cells were then treated with the candidate compounds, and colony forming ability was determined in dose response assays. Colonies were stained with crystal violet and counted after 7 days for ovarian cancer cells and 14 days for diploid fibroblasts. All cell lines were also treated with paclitaxel as a positive control and DMSO $(0.1 \% \mathrm{v} / \mathrm{vol})$ as a negative control.

Mitotic indices. Mitotic index determination is a way to assess quantitatively the number of mitotic events that occur after treatment with candidate compounds [24]. Cancer cells were treated at the IC50 for candidate compounds for 24 hours; diploid fibroblasts were treated for 48 hours to allow for just over a single doubling period for their respective cell lines. All cells were treated with paclitaxel as a positive control and DMSO $(0.1 \% \mathrm{v} / \mathrm{vol})$ as a negative control. Cells were harvested by trypsinization following drug treatment. Media, wash, and trypsin were collected together and centrifuged to pellet the cells. Cells were resuspended in serum free media and treated in $0.4 \% \mathrm{KCl}$ for 10 minutes at $37^{\circ} \mathrm{C}$. Methanol:acetic acid fixative solution $(3: 1, v / v)$ was then added to $2 \%(v / v)$. Subsequent to centrifugation, cells were fixed at room temperature for 20 minutes. Samples of the suspensions were dropped onto glass slides, air dried, and stained with Wright Giemsa solution. Slides were examined under a microscope and at least 200 nuclei were counted for determination of mitotic index.

Apoptosis assays. Determination of apoptosis was determined by the induction of caspase 3 activity, an indicator of apoptosis. This experiment was performed using the Apo-ONE $®$ Homogeneous Caspase-3/7 Assay kit obtained from Promega. Cancer cells were plated in an opaque 96-well plate and treated with a range of compound concentrations for 24 hours. Diploid fibroblasts were treated for 48 hours. All cells were treated with paclitaxel as a positive control and DMSO $(0.1 \% \mathrm{v} / \mathrm{vol})$ as a negative control. The caspase-3/7 substrate rhodamine 
110, bis-(N-CBZ-L-aspartyl-L-glutamyl-L-valyl-L-aspartic acid amide (Z-DEVD-R110) becomes fluorescent after cleavage by caspase 3 . This substrate was added to the samples during the last hour of treatment and fluorescence was measured using a plate reader at $485 \mathrm{~nm} \pm 20$ excitation and $528 \mathrm{~nm} \pm 25$ emission.

Statistical analyses. Statistical analysis was performed by GraphPad Prism 4 software using One-way ANOVA and Dunnett's Post Hoc test for comparison of all experimental compounds against DMSO controls. 

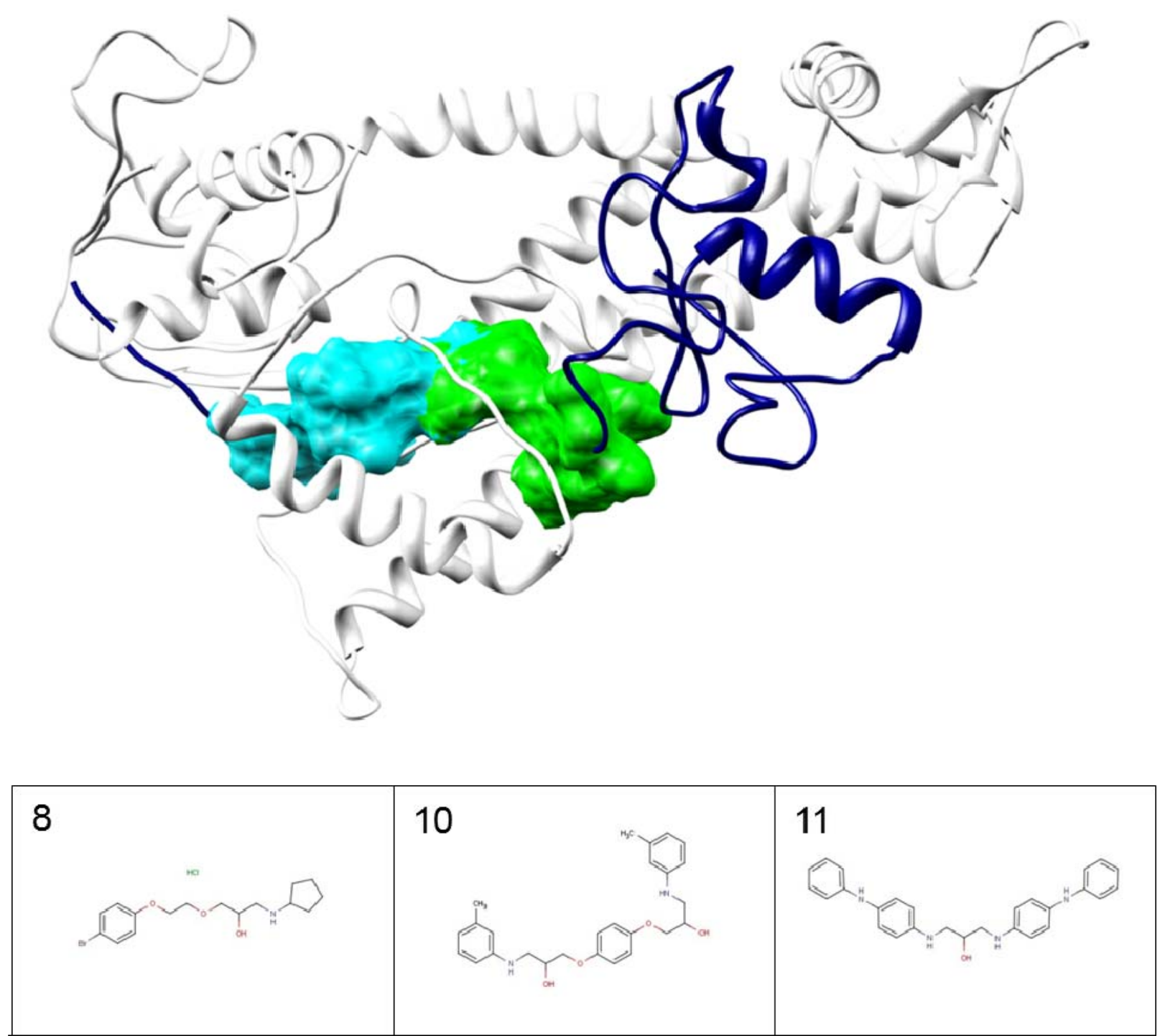

Figure 2. Target binding sites. (Above) Homology structure model of ANAPC2/ANAPC11 interaction showing target sites for drug binding. Sites apc_a (green) and apc_b (turquoise) represent potential target sites for disrupting the binding of ANAPC11 by ANAPC2. (Below) Chemical structures of candidate compounds 8, 10, and 11 selected to target site $b$. 


\section{$\underline{\text { Results }}$}

Visual assessment of compound toxicity. An initial qualitative test of the hypothesis that exposure to the candidate compounds that putatively target the APC/C would cause mitotic arrest displayed the potential for the three tested compounds to induce mitotic arrest and apoptosis in A2780/CP70 ovarian carcinoma cells. A2780/CP70 cells were seeded in a 24 well plate and exposed to varying concentrations of the compounds. The effects of treatment with the compounds were apparent under light microscopy. This experiment showed a dose dependent increase in mitotic and apoptotic cells after a 24 hour exposure of compound 8 . This experiment also tested compounds 10 and 11 at concentrations similar to those used with compound 8 (5-60 $\mu \mathrm{M})$, however a dose dependent increase was not observed over this wide range of concentrations with either compound 10 or 11 (data not shown). A2780/CP70 cells treated with greater than $30 \mu \mathrm{M}$ of compound 10 or greater than $20 \mu \mathrm{M}$ of compound 11 displayed complete obliteration of carcinoma cells. A2780/CP70 cells treated at the lowest concentrations with compound 10 and 11 showed some survival. Therefore, it was decided to repeat the experiment at lower concentrations for compounds 10 and 11 . The data from this experiment showed a similar dose response as was observed in the previous experiment with compound 8 (Figure 3). 


\section{A2780/CP70 Morphology over $24 \mathrm{~h}$ exposure}

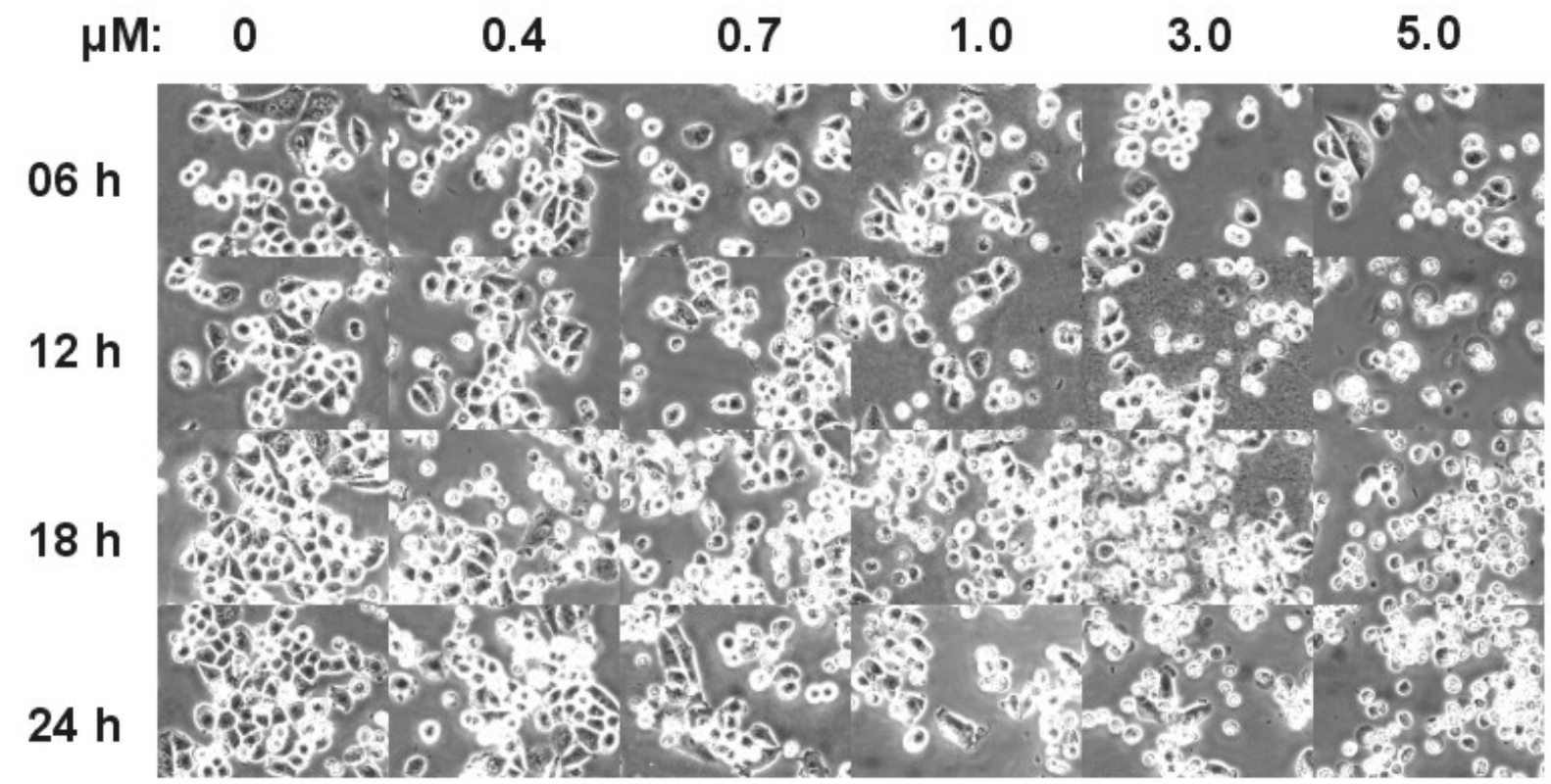

Figure 3. Visual assessment of Compound 10 in A2780/CP70.

Ovarian cancer cells, A2780/CP70, were plated in a 24 well plate and treated with the indicated concentrations of compound 10over a 24 hour period. Pictures were taken at 6 hour intervals. Round, bright mitotic cells can be observed steadily increasing in number as compound concentration increases. Darker, blebbed apoptotic cells appear at the highest drug concentrations. 
The prevalence of bright, round mitotic cells that are indicative of cells undergoing mitosis was observed to increase at a common time point as concentration of the candidate compounds increased. This increase in mitotic cells was also observed to increase over time in wells treated at a particular concentration. A qualitative effect of compound 10 and compound 11 exposures was observed at drug concentrations as low as $400 \mathrm{nM}$, suggesting a difference in the potency of the compounds (Figure 4). The experiment was repeated under the same parameters with a second ovarian carcinoma cell line, SKOV3, and produced similar results (Figure 5). In order to determine whether this cytotoxic effect was observable in normal human fibroblasts, the experiment was repeated with tGM24 diploid fibroblasts at the concentrations observed to kill the cancer cells. Some of the fibroblasts exhibited characteristics of a mitotic delay, but were not completely obliterated as was observed in carcinoma cells. (Figure 6) This finding was consistent with our hypothesis that diploid fibroblasts would be able to recover from prolonged mitotic arrest, and also suggested the potential for a differential toxicity between carcinoma and diploid fibroblasts that would need to be tested further. 
A2780/CP70 Morphology after $12 \mathrm{~h}$ exposure
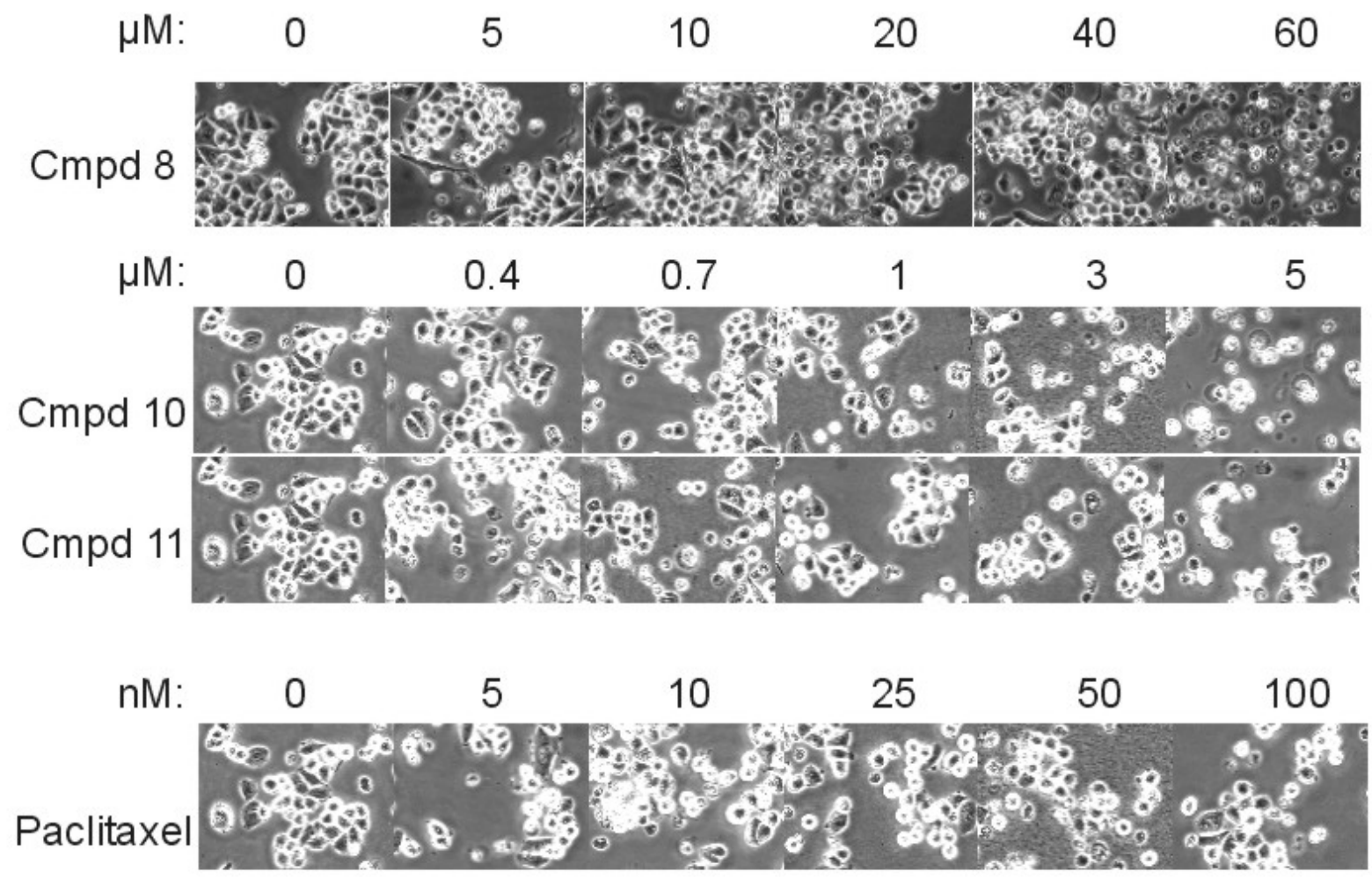

Figure 4. Visual assessment of Compound 8, 10, and 11 in A2780/CP70.

Ovarian cancer cells, A2780/CP70, were plated in a 24 well plate and treated with the indicated concentrations of compounds 8, 10, and 11 over a 24 hour period. Pictures were taken at 6 hour intervals. Round, bright mitotic cells can be observed steadily increasing in number as compound concentration increases. Darker, blebbed apoptotic cells appear at the highest drug concentrations. 
SKOV3 Morphology after $12 \mathrm{~h}$ exposure

$\begin{array}{lllllll}\mu \mathrm{M}: & 0 & 5 & 10 & 20 & 40 & 60\end{array}$
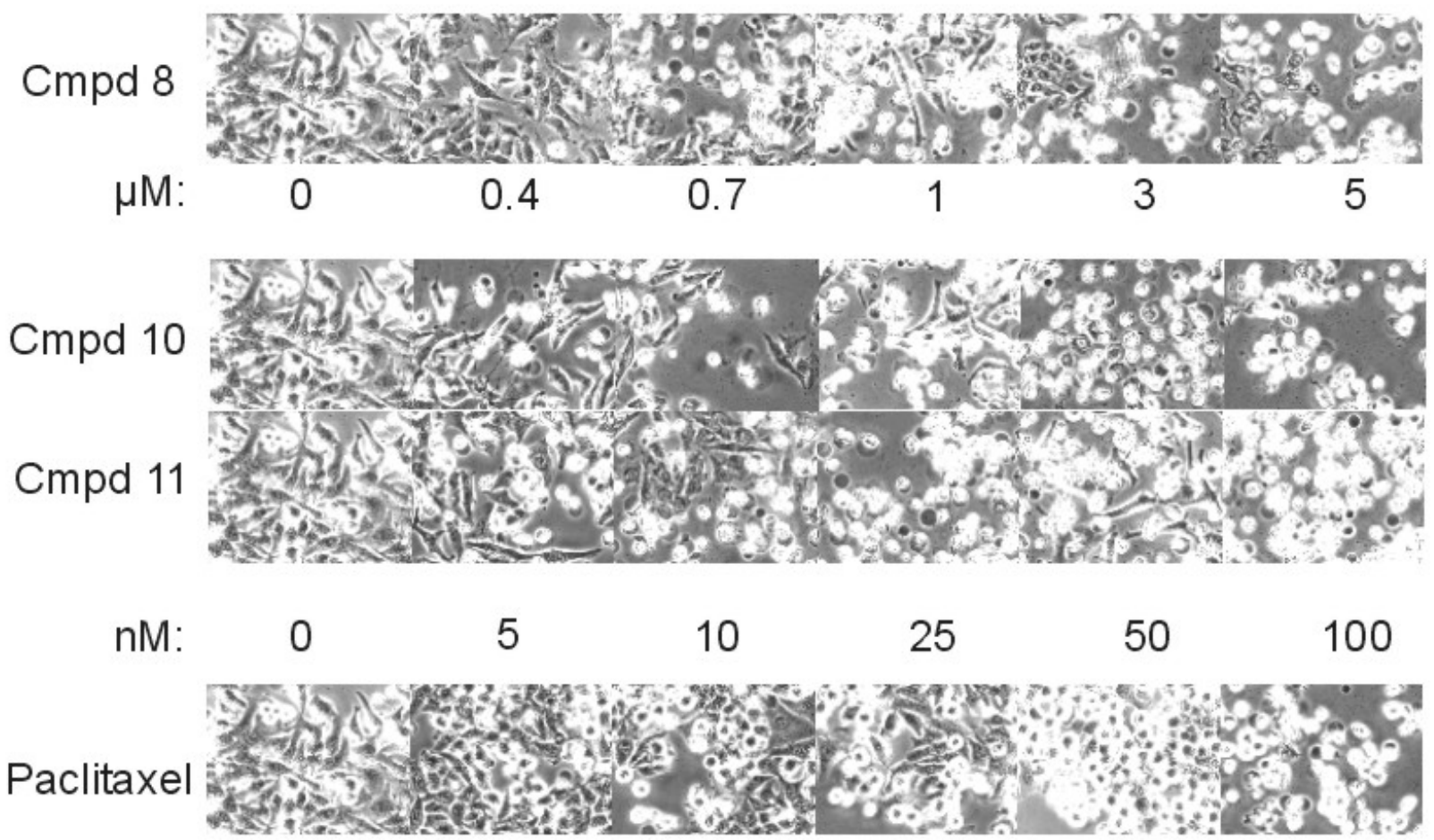

Figure 5. Visual assessment of Compound 8, 10, and 11 in SKOV3.

Ovarian cancer cells, SKOV3, were plated in a 24 well plate and treated with the indicated concentrations of compounds 8, 10, and 11 over a 24 hour period. Pictures were taken at 6 hour intervals. Round, bright mitotic cells can be observed steadily increasing in number as compound concentration increases. Darker, blebbed apoptotic cells appear at the highest drug concentrations. 
tGM24 Morphology after $24 \mathrm{~h}$ exposure
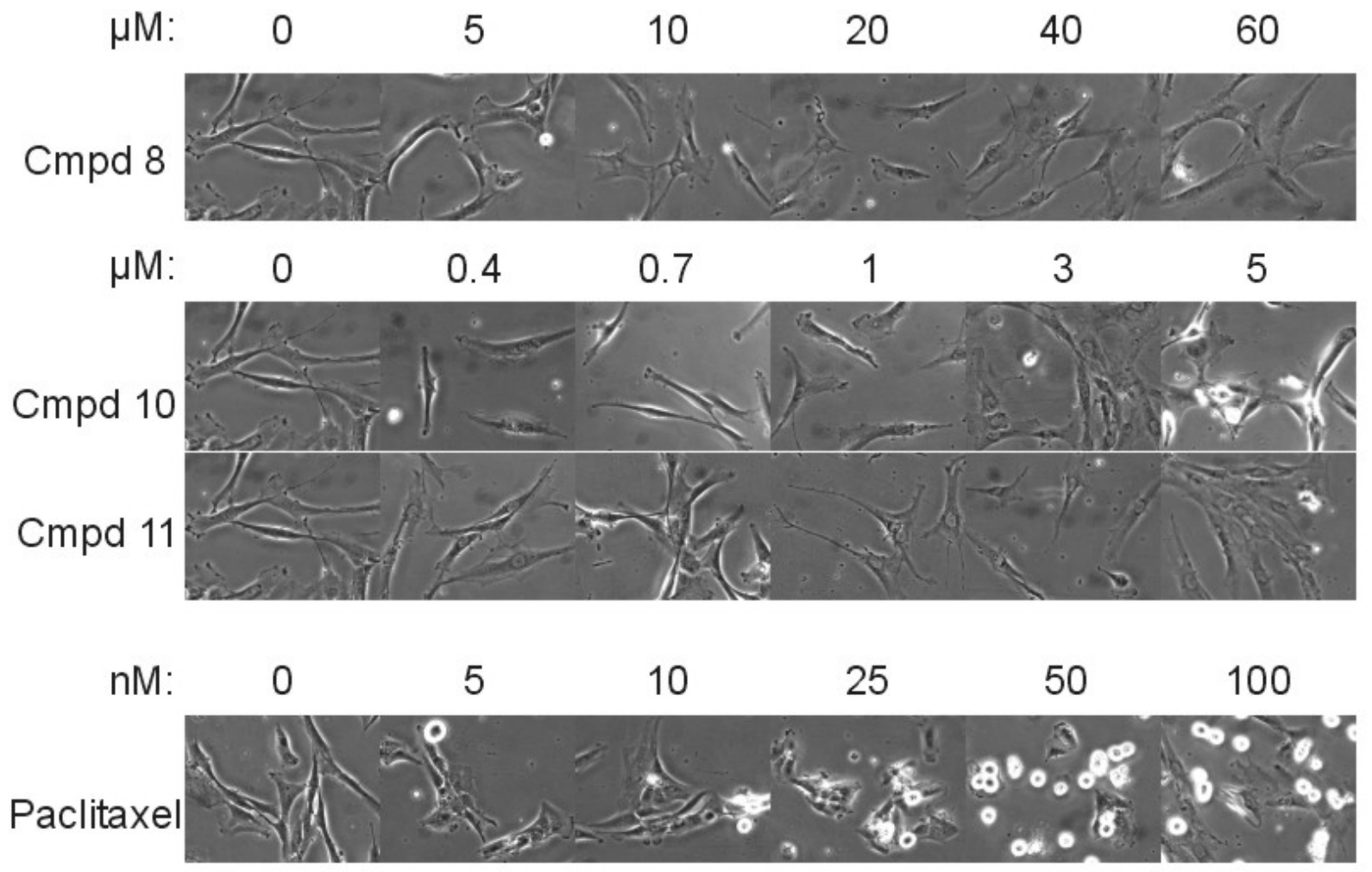

Figure 6. Visual assessment of Compound 8, 10, and 11 in tGM24.

Telomerase immortalized diploid fibroblasts, tGM24, were plated in a 24 well plate and treated with the indicated concentrations of compounds 8, 10, and 11 over a 24 hour period. Pictures were taken at 6 hour intervals. Round, bright mitotic cells can be observed steadily increasing in number as compound concentration increases. Darker, blebbed apoptotic cells are not present at the highest drug concentrations in diploid fibroblasts. 
Cancer cells (A2780/CP70 and SKOV3) exposed to compounds showed a similar increase in the prevalence of darker, blebbed apoptotic cells with respect to concentration and time (Figures 4 and 5). These apoptotic cells were observed in diploid fibroblasts (tGM24) only at the highest compound concentrations tested (Figure 6).

Assessment of compound inhibition of cell growth. It became apparent that future experiments with the compounds would require a consistent baseline concentration with which to treat cells, as well as a quantitative analysis of the observed cytotoxicity. Thus, colony forming assays of the three cell lines were performed. To provide a quantitative assessment of the observed cytotoxicity of the candidate compounds, ovarian cancer cells and fibroblasts were seeded in a 6-well plate, exposed to compounds for a period of 7 to 14 days, and formed colonies were counted. Compounds 10 and 11 were found to be more potent at inhibiting cell growth than compound 8, showing near complete inhibition at $10 \mu \mathrm{M}$ in all cell lines tested (Figures 7, 8 and 9). 


\section{Inhibition of A2780/CP70 Cell Growth}

$$
-\diamond-\operatorname{Cmp} 8 \quad-\circ-\operatorname{Cmp} 10 \quad-\square-\mathrm{Cmp} 11
$$

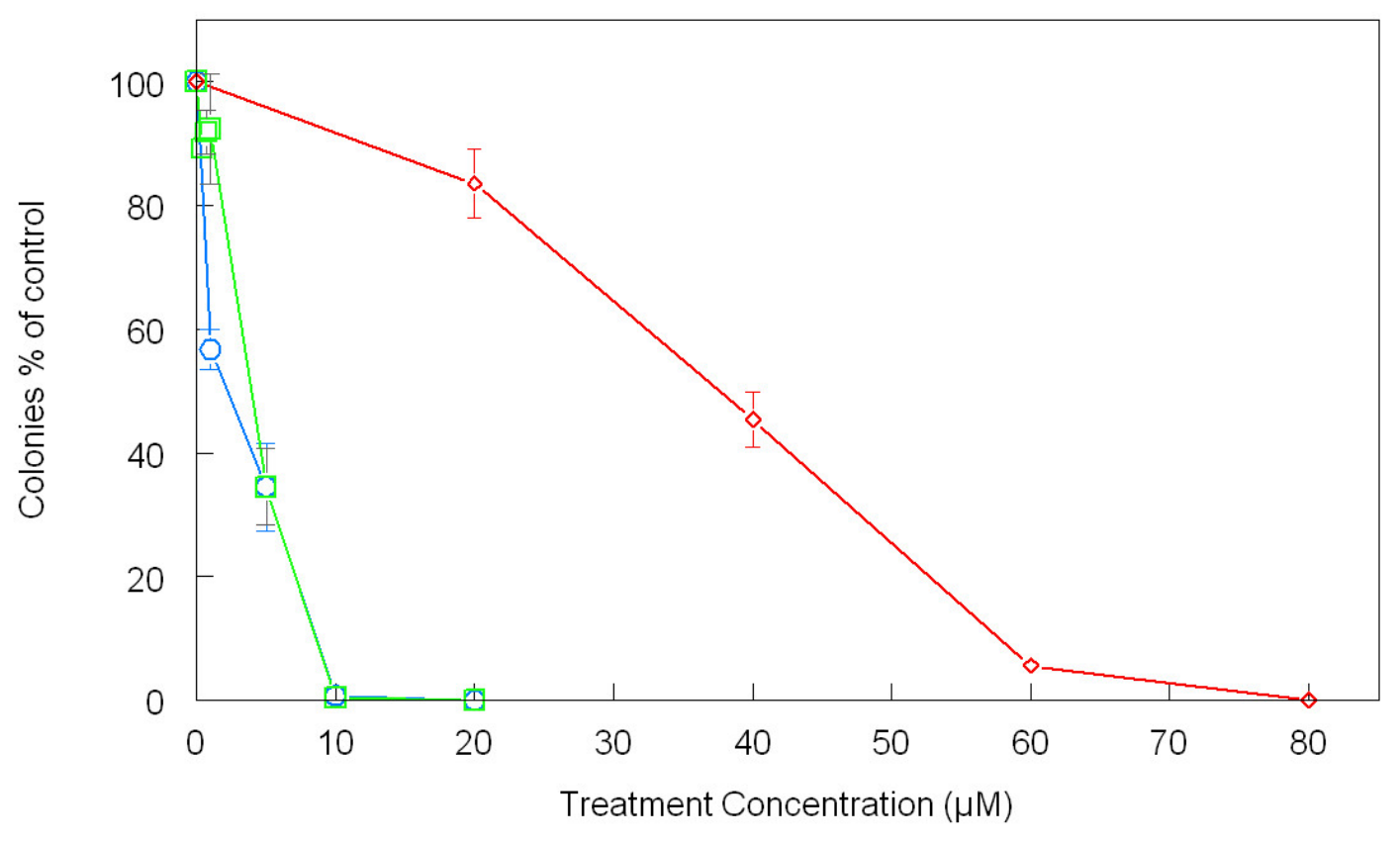

Figure 7. Assessment of compound 8, 10, and 11 inhibition of A2780/CP70 cell growth. Ovarian cancer cells, A2780/CP70, were plated in a 6-well plate at 100 or 500 cells per well, allowed to attach overnight, and then treated with the indicated concentrations. Colonies were stained and counted after 7 days. All compounds completely inhibited ovarian cancer cell proliferation. 


\section{Inhibition of SKOV3 Cell Growth}

$-\diamond-\operatorname{Cmp} 8 \quad-\circ-\mathrm{Cmp} 10 \quad-\square-\mathrm{Cmp} 11$

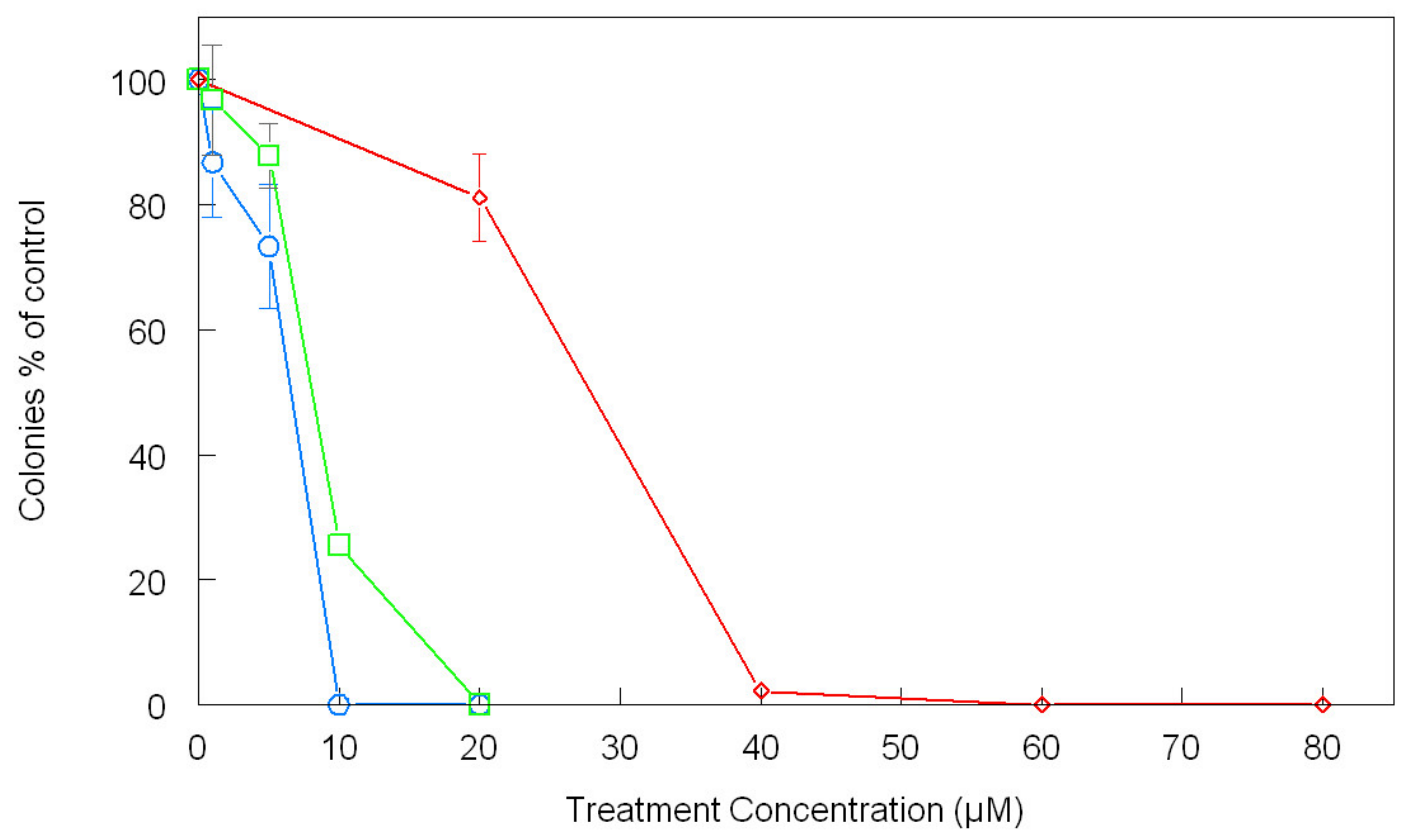

Figure 8. Assessment of compound 8, 10, and 11 inhibition of SKOV3 cell growth. Ovarian cancer cells, SKOV3, were plated in a 6 -well plate at 100 or 500 cells per well, allowed to attach overnight, and then treated with the indicated concentrations. Colonies were stained and counted after 7 days. All compounds completely inhibited ovarian cancer cell proliferation. 


\section{Inhibition of tGM24 Cell Growth}

$$
-\diamond-\operatorname{Cmp} 8 \quad-\circ-\mathrm{Cmp} 10 \quad-\square-\mathrm{Cmp} 11
$$

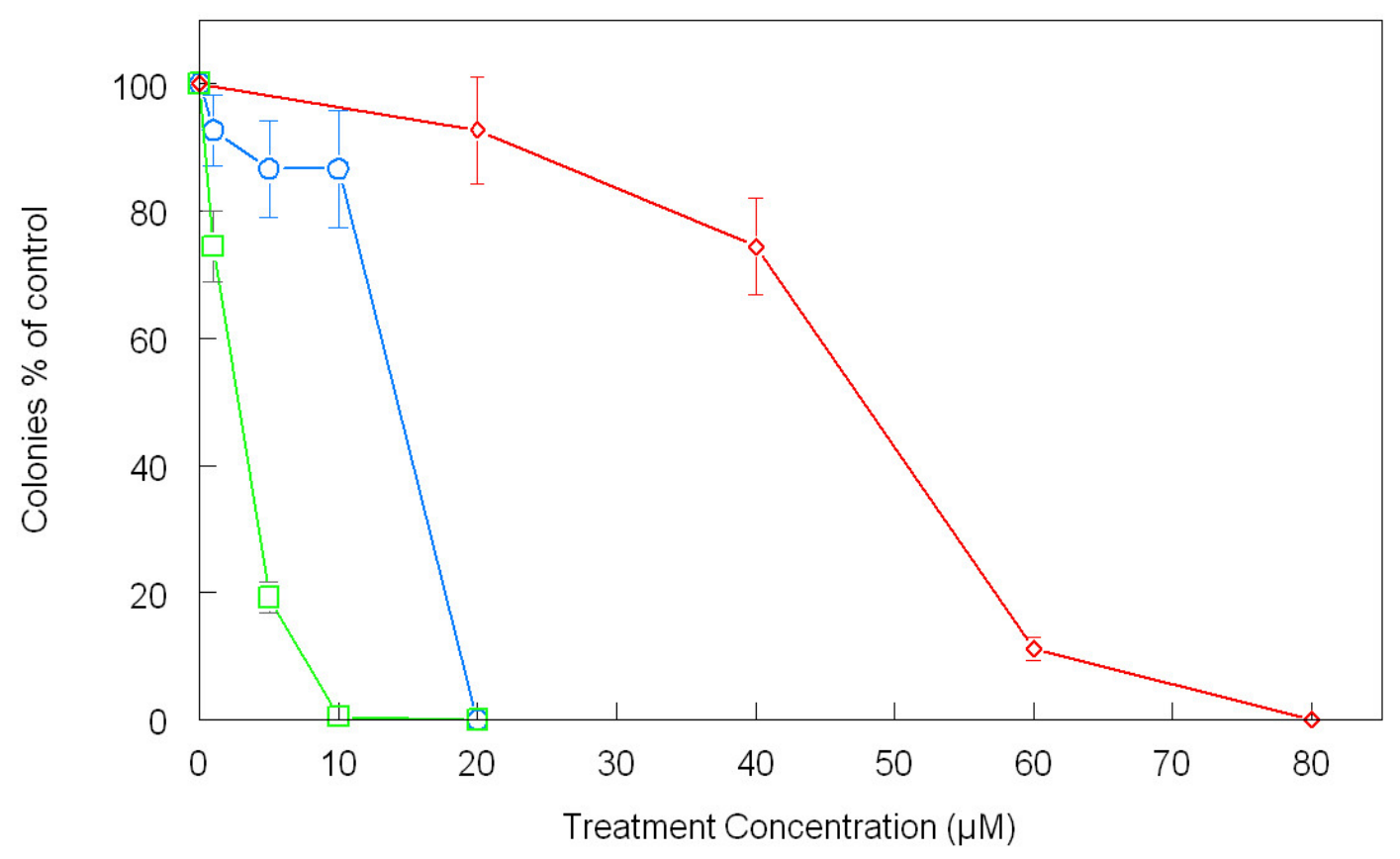

Figure 9. Assessment of compound 8, 10, and 11 inhibition of tGM24 cell growth. Telomerase immortalized diploid fibroblasts, tGM24, were plated in a 6-well plate at 100 or 500 cells per well, allowed to attach overnight, and then treated with the indicated concentrations. Colonies were stained and counted after 14 days. All compounds completely inhibited diploid fibroblast cell proliferation. 
A2780/CP70 cells were slightly more resistant to treatment with compound 8 compared to SKOV3 cells. In all cell lines tested, all compounds were completely efficacious in inhibiting cell growth at the highest concentrations tested. These data also confirmed the relative sensitivity of carcinoma cells to compounds 10 and 11 as compared to compound 8 (Figures 7 and 8). Diploid fibroblasts showed moderate resistance to compound 10 compared to compound 11 (Figure 9). Interestingly, in plates containing diploid fibroblasts treated with the highest concentrations of compound 11, it was still possible to observe individual cells flattened out in the wells at a frequency that seemed consistent with the original plating density, whereas at the same concentrations none of the carcinoma cells remained. These data further supported the observation in the previous experiment that diploid cells were able to recover from exposure to the candidate compounds. Quantitative data from the colony forming assay were analyzed by GraphPad Prism 5 software and IC 50 concentrations were determined. IC50 concentrations of compounds in A2780/CP70 were determined to be $40 \mu \mathrm{M}$ for compound 8, $6 \mu \mathrm{M}$ for compound 10, and $5 \mu \mathrm{M}$ for compound 11 . IC50 concentrations of compounds in SKOV3 were determined to be $31.6 \mu \mathrm{M}$ for compound 8, $5 \mu \mathrm{M}$ for compound 10, and $5 \mu \mathrm{M}$ for compound 11 .

Assessment of mitotic indexes. Armed with a baseline exposure concentration, it began to be possible to assess whether the compounds were actually inducing a mitotic delay. To quantitatively determine the increased incidence of mitotic cells observed previously, mitotic indexes were performed for all cell lines. After determining the approximate cell doubling time for each cell line, carcinoma cells and diploid fibroblasts were treated at IC50 concentrations and mitotic nuclei were counted. All cell lines were treated at the IC50 concentrations determined from the colony forming assay. At IC50 concentrations, all compounds were observed to significantly increase the amount of cells arrested in mitosis as determined by mitotic index in A2780/CP70 and SKOV3 ovarian carcinoma cells (Figures 10 and 11). Diploid fibroblasts also showed a significant increase in mitotic nuclei (Figure 12). 

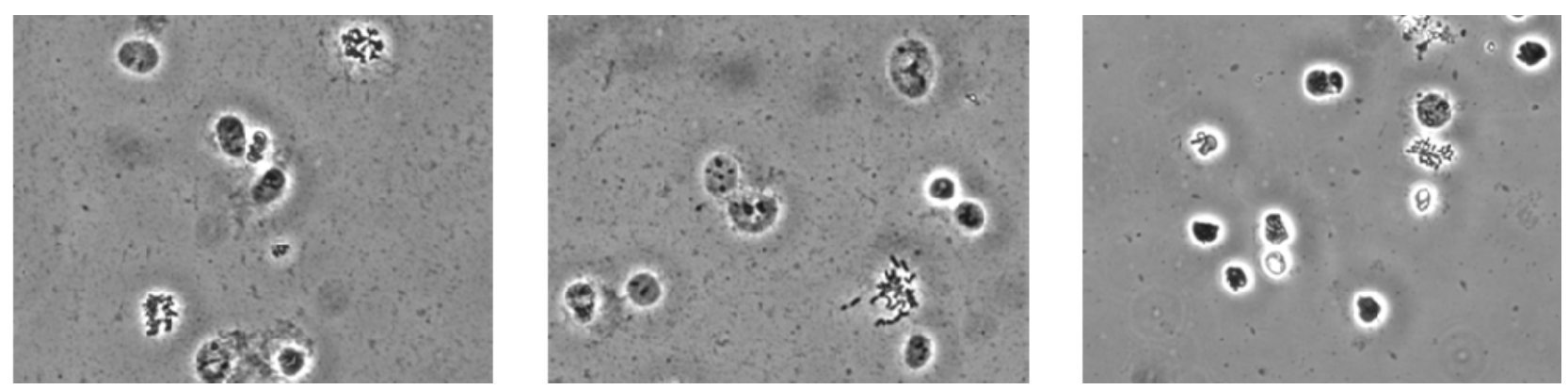

\section{A2780/CP70 Mitotic Index $24 \mathrm{~h}$}

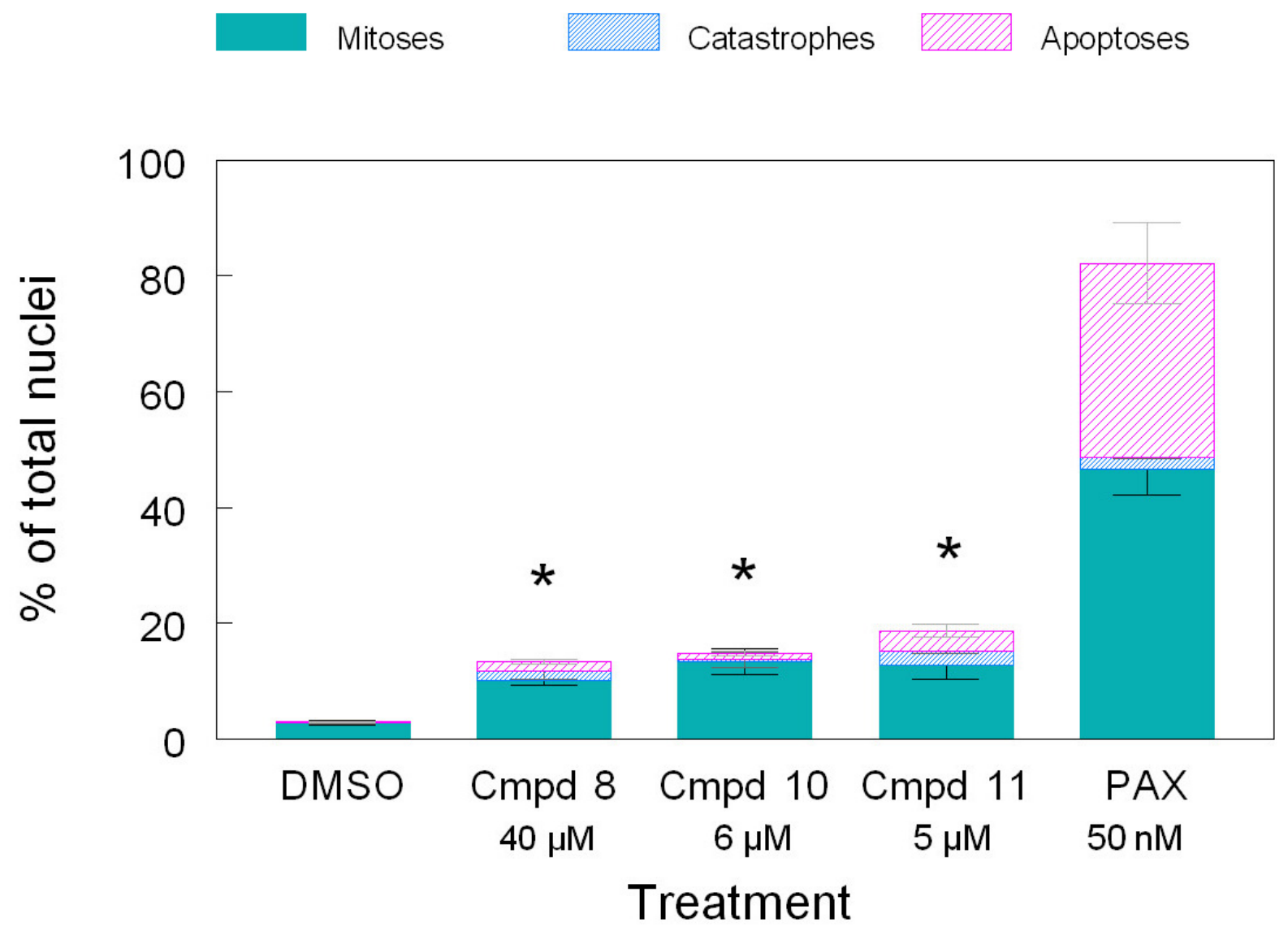

Figure 10. Mitotic index determination in A2780/CP70.

Ovarian cancer cells, A2780/CP70, were treated at IC50 concentrations for compounds 8, 10, and 11 for 24 hours.

Above. Photomicrographs of mitotic spreads. Note mitotic nuclei with condensed chromatin. Below. Quantitation of mitotic index. All compounds induced significant increases in mitotic index as compared to DMSO $(0.1 \% \mathrm{v} / \mathrm{vol})$ treated controls, and were significantly different than paclitaxel controls. $(*=p<0.05)$ 


\section{SKOV3 Mitotic Index $24 \mathrm{~h}$}

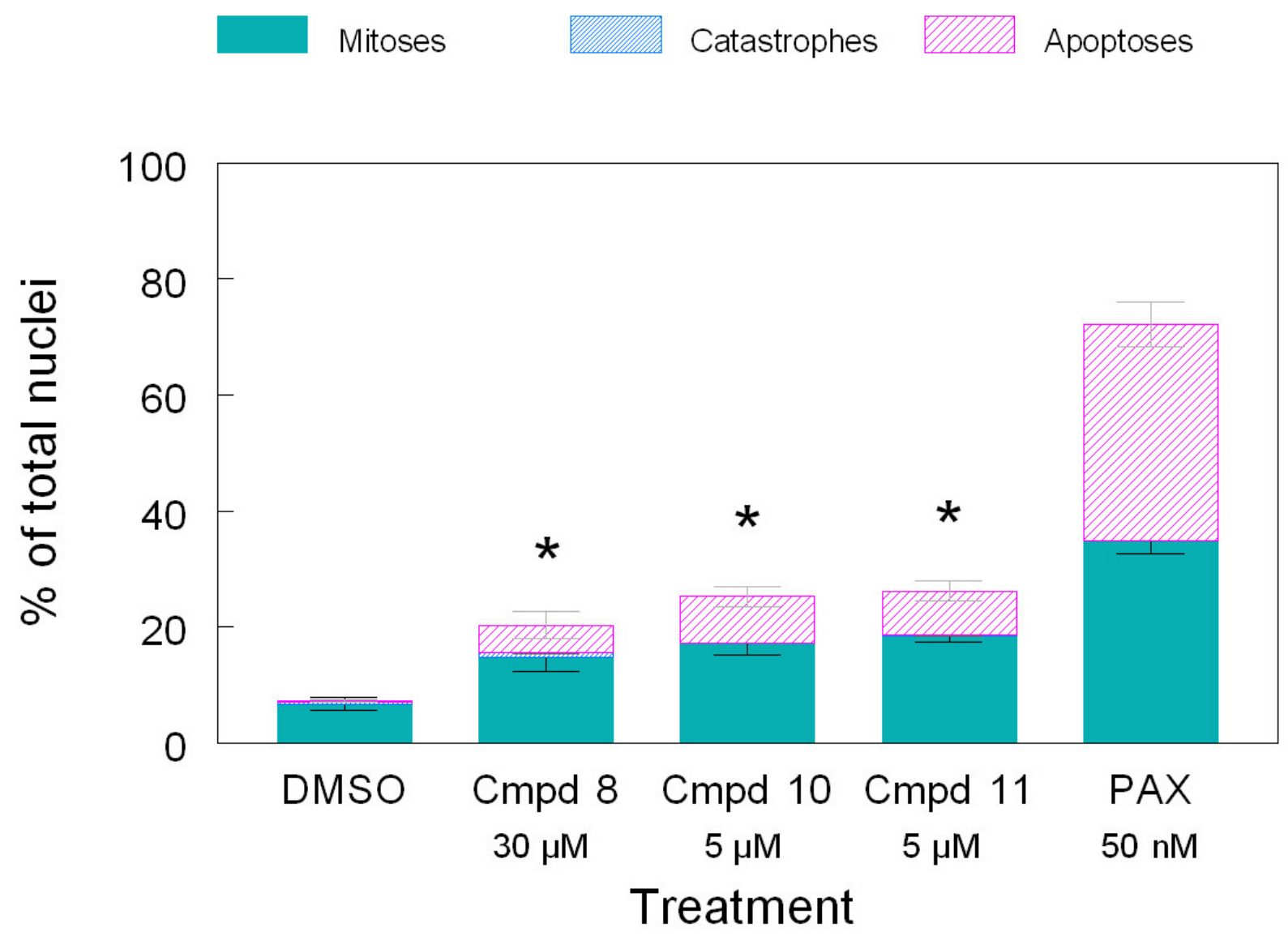

Figure 11. Mitotic index determination in SKOV3.

Ovarian cancer cells, SKOV3, were treated at IC50 concentrations for compounds 8, 10, and 11 for 24 hours. All compounds induced significant increases in mitotic index as compared to DMSO $(0.1 \% \mathrm{v} / \mathrm{vol})$ treated controls, and were significantly different than paclitaxel controls. $\left({ }^{*}=p<0.05\right)$ 


\section{tGM24 Mitotic Index $48 \mathrm{~h}$}

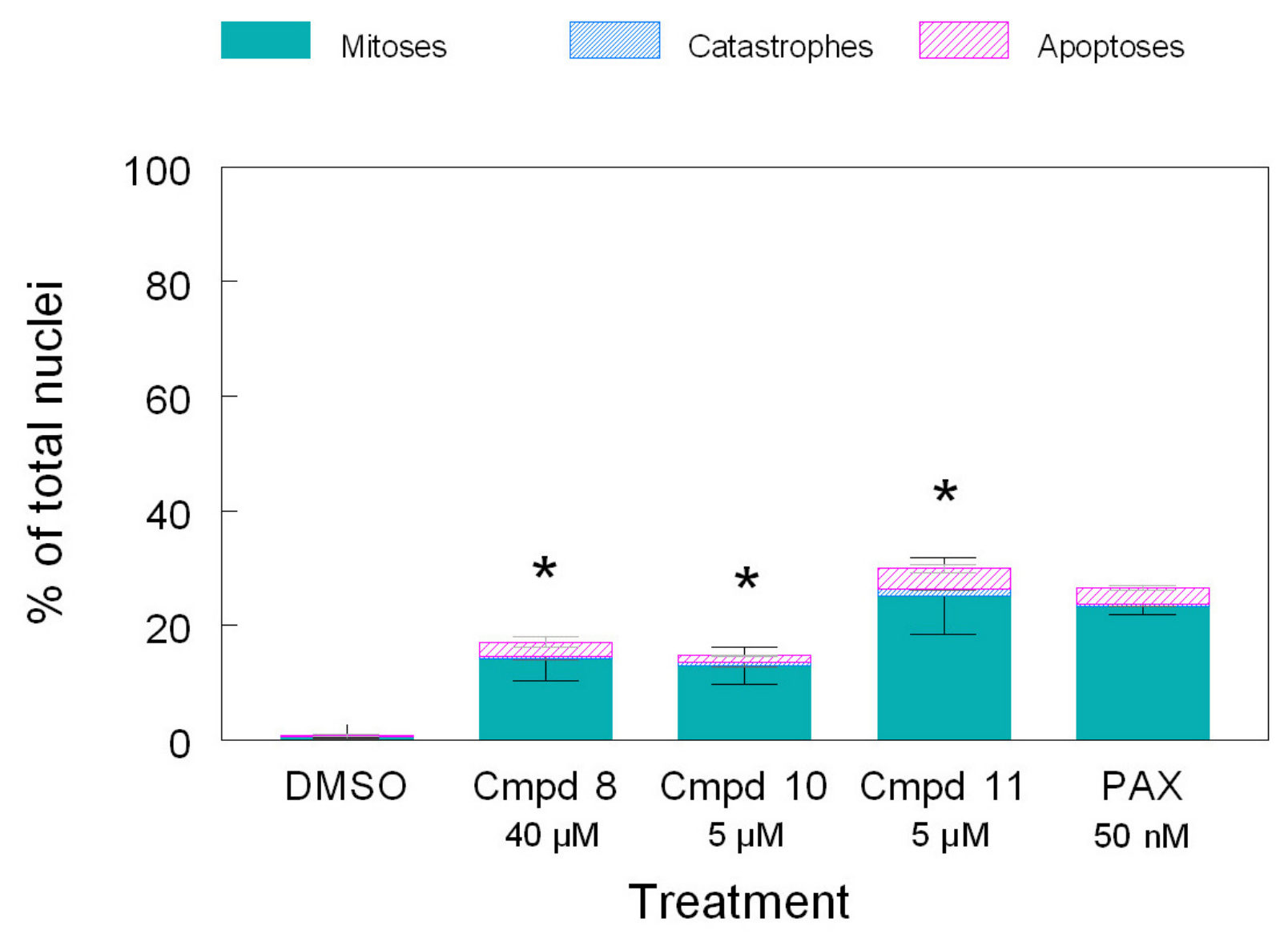

Figure 12. Mitotic index determination in tGM24.

Telomerase immortalized diploid fibroblasts, tGM24, were treated at IC50 concentrations for compounds 8,10 , and 11 for 48 hours. All compounds induced significant increases in mitotic index as compared to DMSO $(0.1 \% \mathrm{v} / \mathrm{vol})$ treated controls, but were not significantly different from paclitaxel controls. $(*=p<0.05)$ 
In A2780/CP70 ovarian carcinoma cells, Compound 10 induced the greatest increase in mitotic nuclei to $13 \%$ of nuclei counted. Treatment with Compound 11 induced a similar increase of mitotic nuclei to 13\%. Treatment with Compound 8 resulted in 10\% of nuclei in mitosis (Figure 8). In SKOV3 ovarian carcinoma cells, Compound 11 induced the greatest increase in mitotic nuclei to $19 \%$ of nuclei counted. Treatment with Compound 10 resulted in $17 \%$ mitotic nuclei. Treatment with Compound 8 resulted in an increase of mitotic nuclei to 15\% (Figure 9). In tGM24 diploid fibroblasts, treatment with Compound 11 induced the greatest increase in mitotic nuclei to $25 \%$ nuclei counted. This increase was greater than mitotic nuclei counted after treatment with the $50 \mathrm{nM}$ Paclitaxel control (23\%). Treatment with Compound 8 induced the next greatest increase in mitotic nuclei to $14 \%$ counted. Treatment with Compound 10 resulted in an increase of mitotic nuclei to $13 \%$ counted (Figure 10). The values for mitotic nuclei counted for all compounds tested were determined to be significantly different as determined by One-way ANOVA and Dunnett's Post Hoc test against DMSO controls.

While a precise duration of mitotic arrest was not determined, compounds 10 and 11 were shown to be more potent in their ability to induce mitotic arrest as compared to compound 8. However, at IC50 concentrations, the three candidate compounds did not show a significant difference relative to each other in their ability to induce mitotic arrest. This could lend support to the hypothesis that the compounds are acting on the same target. There was not a significant increase in the prevalence of nuclei associated mitotic catastrophe, however qualitatively there appeared to be more observable instances of mitotic catastrophe in cells treated with candidate compounds than with DMSO. This result is not completely unexpected as mitotic catastrophe is expected in cells lacking a functional SAC, and all cell lines tested are believed to have a functional spindle assembly checkpoint. Interestingly, there was not a noticeable difference in the prevalence of characteristically apoptotic nuclei in carcinoma cells as compared to paclitaxel controls. 
Assessment of caspase 3 activity. It became important to determine whether the cytotoxic effects of the compounds observed in earlier experiments were indeed due to induction of the apoptotic pathway. In order to determine that compounds were causing cells to activate the apoptotic pathway, caspase-3/7 activation was quantitatively measured using an Apo-ONE Homogenous Caspase-3/7 Assay. Caspase 3 and 7 activity, indicators of apoptosis, was measured via the addition of a fluorescent substrate to wells containing either ovarian carcinoma or diploid fibroblasts that had been treated with compounds. This substrate contains peptide sequences that are cleaved by caspase 3 and expose a leaving group that is highly aromatic and becomes intensely fluorescent when excited at $499 \mathrm{~nm}$. All cell lines were treated at the determined IC50 concentrations for the test compounds. Compounds 10 and 11 resulted in a significant increase in caspase activation in A2780/CP70 and SKOV3 ovarian carcinoma cells relative to DMSO controls (Figures 13 and 14). Caspase activation was shown to be highest in carcinoma cells treated with compound 11, which showed activation in a dose dependent manner. Compound 10 also showed activation in ovarian carcinoma cells. Compound 8, the least potent of the three candidate compounds tested, showed very little increase in caspase activation relative to DMSO controls (Figures 13 and 14). Diploid fibroblasts did not show a significant increase in caspase activation, consistent with prior data reporting that fibroblasts were able to survive exposure to compounds (Figure 15). 


\section{A2780/CP70 Caspase activity}

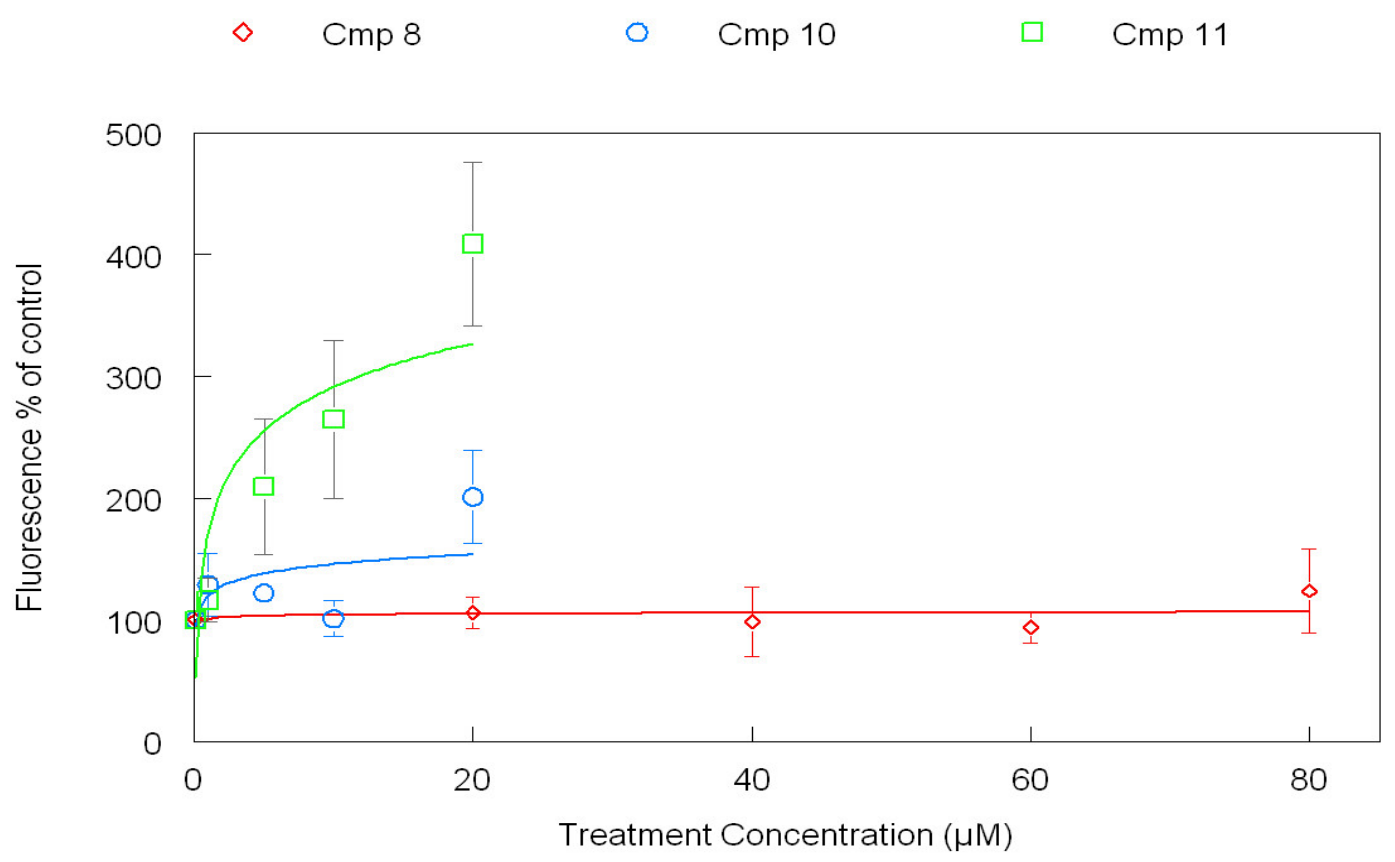

Figure 13. Assessment of caspase 3 activity in A2780/CP70.

Ovarian cancer cells, A2780/CP70, were plated in a 96-well plate and treated at the indicated concentrations for 24 hours. During the last hour of incubation, fluorescent caspase 3 substrate was added to the media. Data are reported as RFU relative to DMSO $(0.1 \% \mathrm{v} / \mathrm{vol})$ treated controls vs. concentration. 


\section{SKOV3 Caspase activity}

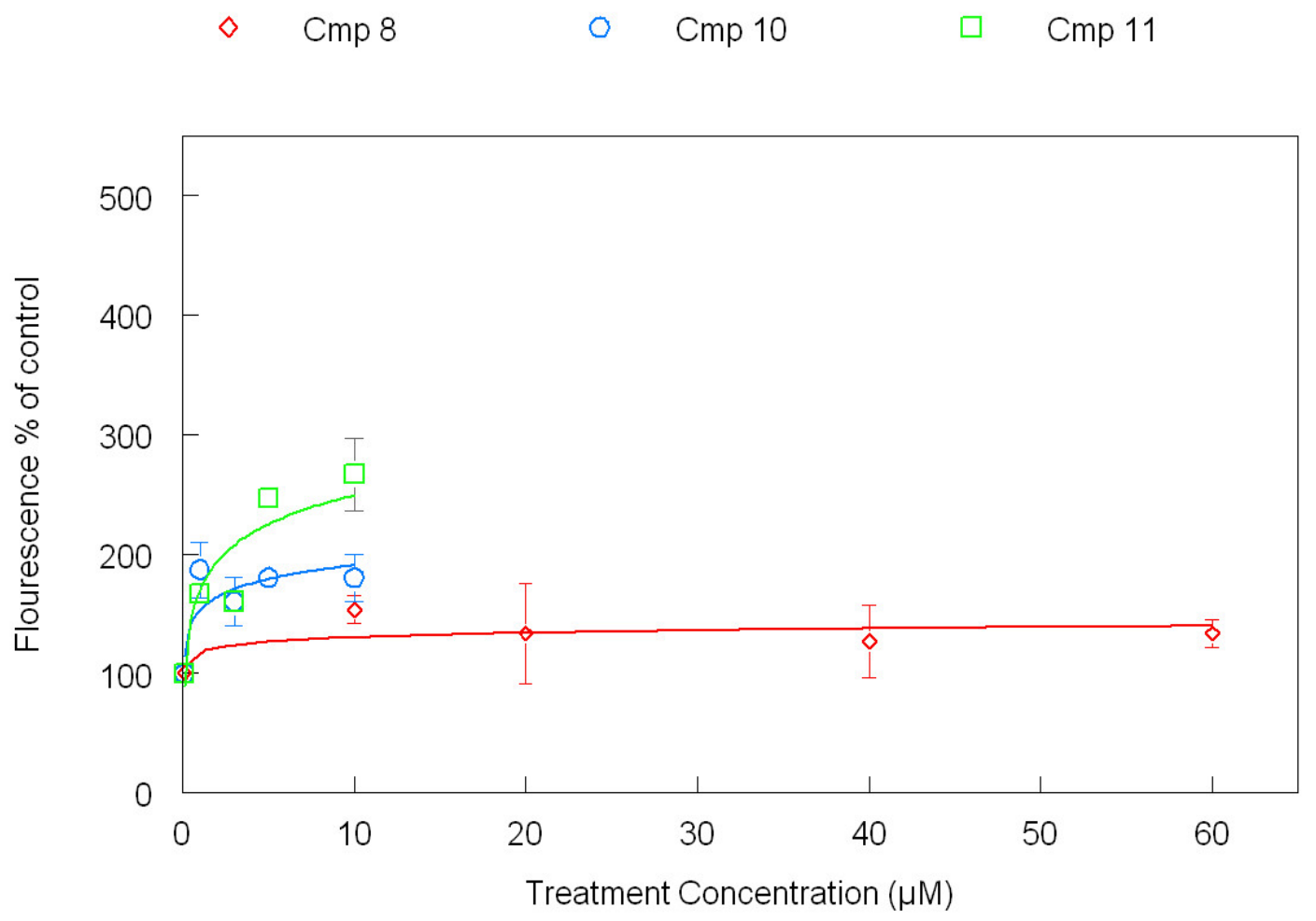

Figure 14. Assessment of caspase 3 activity in SKOV3.

Ovarian cancer cells, SKOV3, were plated in a 96-well plate and treated at the indicated concentrations for 24 hours. During the last hour of incubation, fluorescent caspase 3 substrate was added to the media. Data are reported as RFU relative to DMSO $(0.1 \% \mathrm{v} / \mathrm{vol})$ treated controls vs. concentration. 


\section{tGM24 Caspase activity}

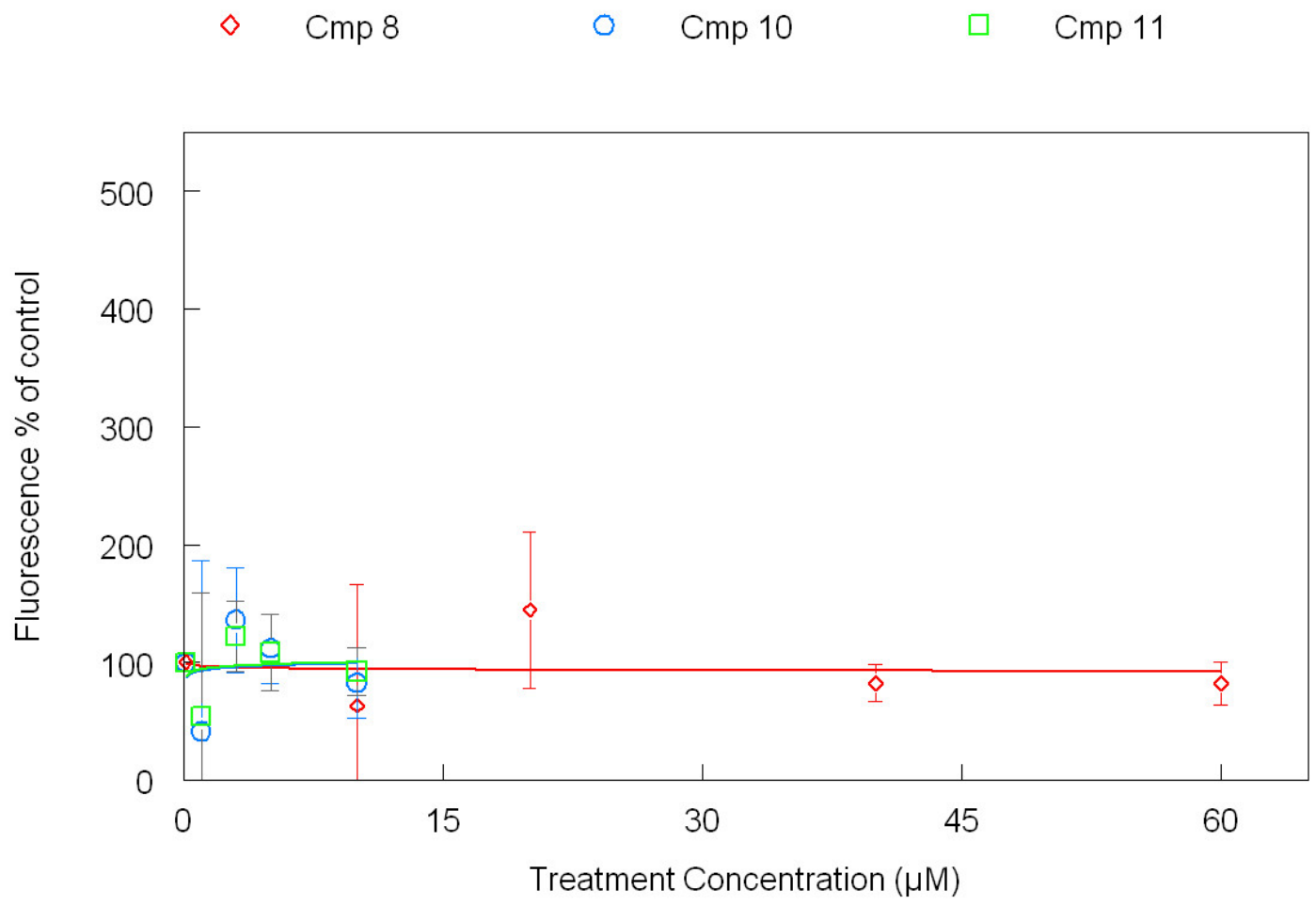

Figure 15. Assessment of caspase 3 activity in tGM24.

Telomerase immortalized diploid fibroblasts, tGM24, were plated in a 96-well plate and treated at the indicated concentrations for 48 hours. During the last hour of incubation, fluorescent caspase 3 substrate was added to the media. Data are reported as RFU relative to DMSO $(0.1 \% \mathrm{v} / \mathrm{vol})$ treated controls vs. concentration. 
Expression and purification of recombinant ANAPC2 protein. Cumulatively, the data gained from these experiments suggest that compounds 10 and 11 are very potent and efficacious drugs in inducing mitotic arrest and apoptosis in ovarian cancer in vitro. Further, these compounds show differential toxicity as diploid fibroblasts are able to survive treatment. While these data are promising, it remains to be determined if these compounds are indeed binding and inhibiting their intended target.

In order to determine whether the candidate compounds are binding to their intended target and test the hypothesis that the structure of the compounds affects their binding affinity for ANAPC2, we have attempted to express a recombinant human ANAPC2 in a pQE-30 vector in Escherichia coli with a pREP4 repressor plasmid. This recombinant protein contains a $6 \mathrm{X}$ His tag on the $\mathrm{N}$-terminus of the protein sequence. Unfortunately, while it is possible the protein has been expressed, purification has proven problematic as there appeared to be non-specific protein binding to the Ni-NTA agarose beads that were supposed to bind the His tagged protein. This could be due to protein folding over the His tag, in which case placing the His tag on the Cterminus as well as the $\mathrm{N}$-terminus may facilitate binding. Alternatively, it could be that the protein is not being expressed. This hypothesis is supported by DNA sequencing that identified a possible single nucleotide deletion just after the start sequence of the pQE30 plasmid. 


\section{Discussion}

This thesis examines three small molecules targeting the Anaphase Promoting Complex/Cyclosome for efficacy in inducing mitotic arrest and apoptosis in ovarian cancer cells. Survival rates for those afflicted with later stage ovarian cancer have shown little improvement over the last two decades. This is in part due to the complexity of the various subtypes of ovarian cancer as well as difficulty in procuring early diagnosis. Current treatment involves surgery combined with the peritoneal administration of chemotherapeutics such as cisplatin and paclitaxel. Resistance to cisplatin and paclitaxel remains a difficult problem to overcome in ovarian cancer treatment [25]. Ovarian cancer develops resistance to paclitaxel through a variety of mechanisms including drug efflux, mutant tubulins, and non-functional SAC [15]. In order to combat the emergence of drug resistance to current therapies, it is important to search for new treatments and novel drug targets that can show efficacy in a variety of different cell types.

As the master regulator of cell cycle progression, the APC/C provides potential as a novel target for anti-cancer therapy and may prove to provide a promising avenue of treatment for cancers which are resistant to microtubule stabilizing drugs such as paclitaxel. Inhibition of the APC/C may result in stabilization of proteins whose degradation is essential for progression from the metaphase to anaphase transition such as securin and mitotic cyclin B1. Indeed, CDC20 depletion mediated by siRNA knockdown results in prolonged mitotic arrest and apoptosis in cancer cells [26].This effect was also observed to induce mitotic arrest in cancer cells that lacked a functioning spindle assembly checkpoint, indicating the potential for inhibition of the $\mathrm{APC} / \mathrm{C}$ and its co-activators as potential chemotherapeutic targets.

The approach of using a small molecule to prevent the binding of the co-activators of the APC/C, CDC20 and CDH1, has been demonstrated in the development of tosyl-L-arginine methyl ester (TAME) [21]. This molecule was modified to be cell permeable and tested in HeLa cells. Treatment of cells with this drug resulted in a prolonged mitotic arrest and death response 
that was independent of microtubule disruption. However, TAME appeared to be highly dependent upon expression levels of CDC20. The SAC normally sequesters CDC20 prior to proper chromosome alignment. When the SAC was knocked down, cancer cells were able to recover from the mitotic delay and survive treatment. This study demonstrates the viability of targeting the APC/C to induce mitotic arrest, but also demonstrates that the site which TAME targets may not be affective in cells lacking a functional SAC.

Analysis of the APC/C structure yielded two other potential target sites for compounds to bind to the APC/C [21]. These small molecule compounds, selected from a list of fifty candidates, were designed to bind to the ANAPC2 subunit of the APC/C and thereby inhibit the binding of the ANAPC11 subunit to the complex. Inhibition of the formation of this catalytic component of the APC/C should disrupt the ability of the APC/C to ubiquitinate its protein substrate targets. The data presented in this thesis demonstrate the ability of these compounds to induce significant mitotic arrest in cisplatin resistant ovarian cancer cells. Compounds 10 and 11 showed the greatest ability to induce mitotic arrest and cell death, and compound 11 induced apoptosis in a dose dependent manner. This difference in potency could be accounted for in either the chemical structure of the compounds and their binding affinity of the ANAPC2 binding domain, or in the possibility of off-target binding. The similar efficacy of the compounds in inducing mitotic arrest at their IC50 concentrations lends support to the hypothesis that the compounds are affecting the same target.

It remains to be empirically determined whether the compounds are binding their intended target. This can be assessed by expressing and purifying ANAPC2 and performing a thermal denaturation assay. Unfortunately, obtaining purified ANAPC2 using a polyhistidine tag has proven to be an elusive endeavor. Due to the difficulties faced with purifying the ANAPC2 protein, in the future the ANAPC2 DNA sequence can be excised from its current plasmid vector using restriction enzymes and ligated to a different expression system. The Intein-Mediated Purification with an Affinity Chitin-binding Tag (IMPACT) protein purification system provides 
another viable avenue for expressing and purifying ANAPC2 protein. This system utilizes a selfcleaving peptidase element, intein, fused to a chitin-binding domain. This element can be placed on either the C-terminus or N-terminus of the amino acid sequence. After expression, cell lysate can be run through a chitin column to selectively bind proteins with the chitin-binding domain from other cell materials. Once bound to the column, the peptidase activity of intein is induced and releases the ANAPC2 protein from its tag. Purified ANAPC2 protein can then be used to analyze candidate compound binding affinity through fluorescent thermal denaturation assay. This will allow the determination of other compounds from the list of candidates that bind strongly to the ANAPC2 subunit, providing more opportunities for testing.

In addition to this mechanistic study, there remain further avenues to establishing the efficacy of compounds in SAC deficient (paclitaxel-resistant) cell lines. Preliminary data obtained from the visual assessment of toxicity in SAC-deficient malignant melanoma (SK-MEL28) has shown a similar increase in mitotic and apoptotic cells after treatments with compound 8. This suggests that cells resistant to paclitaxel may also be susceptible to other tested candidate compounds. Transformation of ovarian carcinoma cell lines to paclitaxel resistance can be facilitated by knockdown of SAC components such as BUBR1 or MAD2. In addition to mitotic index, it will be possible to assess the amount of cells that arrest in mitosis and those that arrest in the pseudo-G1 phase through flow cytometry. We have developed a working protocol for this assay utilizing 7-AAD staining for total DNA content and FITC-conjugated antibromodeoxyuridine to stain newly synthesized DNA.

With data provided from these new experiments, it is possible to select the most promising candidates for further modification to provide efficacy at nanomolar ranges. At these pharmacologically viable concentrations, these compounds could act as effective tools in the fight against ovarian cancer. If it is confirmed that the candidate compounds are binding to their protein target, then these data identify that the APC/C is a viable target for future anti-cancer treatments and that the candidate compounds tested may be developed into effective 
chemotherapeutics. Further, these compounds could be used in conjunction with other drugs that have shown efficacy in treatment of ovarian cancer, such as paclitaxel, or compounds that inhibit the function of the APC/C, such as TAME. As both of those treatments are dependent upon a functional SAC, the compounds examined in this thesis may be developed to be a clinically relevant solution to this problem if further testing supports that they are SAC independent. Hopefully, this could lessen the difficulty of treating drug resistant ovarian cancer and ultimately lead to a better quality of life and diminish the suffering of those afflicted with the disease.

\section{Acknowledgments}

This research was supported in part by the University of Louisville Cancer Education Program $\mathrm{NIH} / \mathrm{NCl}$ grant R25-CA134283 and a Basic Research Grant from the University of Louisville School of Medicine. 


\section{Bibliography}

1. Siegel, R., D. Naishadham, and A. Jemal, Cancer statistics, 2012. CA Cancer J Clin, 2012. 62(1): p. 10-29.

2. Goff, B.A., et al., Ovarian carcinoma diagnosis. Cancer, 2000. 89(10): p. 2068-75.

3. Das, P.M. and R.C. Bast, Jr., Early detection of ovarian cancer. Biomark Med, 2008. 2(3): p. 291-303.

4. Smith, L.H., et al., Ovarian cancer: can we make the clinical diagnosis earlier? Cancer, 2005. 104(7): p. 1398-407.

5. Prat, J., Ovarian carcinomas: five distinct diseases with different origins, genetic alterations, and clinicopathological features. Virchows Arch, 2012. 460(3): p. 23749.

6. Gilks, C.B. and J. Prat, Ovarian carcinoma pathology and genetics: recent advances. Hum Pathol, 2009. 40(9): p. 1213-23.

7. Hogberg, T., et al., A systematic overview of chemotherapy effects in ovarian cancer. Acta Oncol, 2001. 40(2-3): p. 340-60.

8. van der Burg, M.E., et al., The effect of debulking surgery after induction chemotherapy on the prognosis in advanced epithelial ovarian cancer. Gynecological Cancer Cooperative Group of the European Organization for Research and Treatment of Cancer. N Engl J Med, 1995. 332(10): p. 629-34.

9. Eitan, R., et al., Clinical Course of Patients Treated for Advanced Ovarian Carcinoma without Surgical Intervention. PLoS One, 2013. 8(1): p. e55645.

10. du Bois, A., et al., 2004 consensus statements on the management of ovarian cancer: final document of the 3rd International Gynecologic Cancer Intergroup Ovarian Cancer Consensus Conference (GCIG OCCC 2004). Ann Oncol, 2005. 16 Suppl 8: p. viii7-viii12.

11. Armstrong, D.K., et al., Intraperitoneal cisplatin and paclitaxel in ovarian cancer. $\mathbf{N}$ Engl J Med, 2006. 354(1): p. 34-43.

12. Sudo, T., et al., Dependence of paclitaxel sensitivity on a functional spindle assembly checkpoint. Cancer Res, 2004. 64(7): p. 2502-8.

13. De Antoni, A., et al., The Mad1/Mad2 complex as a template for Mad2 activation in the spindle assembly checkpoint. Curr Biol, 2005. 15(3): p. 214-25.

14. Milross, C.G., et al., Relationship of mitotic arrest and apoptosis to antitumor effect of paclitaxel. J Natl Cancer Inst, 1996. 88(18): p. 1308-14.

15. Giannakakou, P., et al., Paclitaxel-resistant human ovarian cancer cells have mutant beta-tubulins that exhibit impaired paclitaxel-driven polymerization. J Biol Chem, 1997. 272(27): p. 17118-25.

16. Lindon, C., Control of mitotic exit and cytokinesis by the APC/C. Biochem Soc Trans, 2008. 36(Pt 3): p. 405-10.

17. Manchado, E., M. Eguren, and M. Malumbres, The anaphase-promoting complex/cyclosome (APC/C): cell-cycle-dependent and -independent functions. Biochem Soc Trans, 2010. 38(Pt 1): p. 65-71.

18. Stemmann, O., et al., Dual inhibition of sister chromatid separation at metaphase. Cell, 2001. 107(6): p. 715-26.

19. Petersen, B.O., et al., Cell cycle- and cell growth-regulated proteolysis of mammalian CDC6 is dependent on APC-CDH1. Genes Dev, 2000. 14(18): p. 233043. 
20. Penas, C., V. Ramachandran, and N.G. Ayad, The APC/C Ubiquitin Ligase: From Cell Biology to Tumorigenesis. Front Oncol, 2011. 1: p. 60.

21. Taylor, B.F., Arsenite Inhibition Of Mitotic Progression, in Pharmacology and Toxicology2007, University of Louisville: Louisville, Kentucky.

22. Castedo, M., et al., Cell death by mitotic catastrophe: a molecular definition. Oncogene, 2004. 23(16): p. 2825-37.

23. Porter, P.C., et al., Telomerase-immortalized human fibroblasts retain UV-induced mutagenesis and p53-mediated DNA damage responses. DNA Repair (Amst), 2006. 5(1): p. 61-70.

24. States, J.C., et al., Arsenite disrupts mitosis and induces apoptosis in SV40transformed human skin fibroblasts. Toxicol Appl Pharmacol, 2002. 180(2): p. 8391.

25. Perego, P., et al., Ovarian cancer cisplatin-resistant cell lines: multiple changes including collateral sensitivity to Taxol. Ann Oncol, 1998. 9(4): p. 423-30.

26. Huang, H.C., et al., Evidence that mitotic exit is a better cancer therapeutic target than spindle assembly. Cancer Cell, 2009. 16(4): p. 347-58. 